\title{
Interactions of a Polyanion with a Cationic Micelle: Comparison of Monte Carlo Simulations with Experiment ${ }^{\dagger}$
}

\author{
A. Laguecir, ${ }^{\ddagger}$ S. Stoll, ${ }^{*}$, G. Kirton, ${ }^{\S}$ and P. L. Dubin ${ }^{\S}$ \\ Analytical and Biophysical Environmental Chemistry (CABE), Department of Inorganic, Analytical and Applied \\ Chemistry, University of Geneva, Sciences II, 30 quai E. Ansermet, CH-1211 Geneva 4, Switzerland, and \\ Department of Chemistry, Indiana-Purdue University, Indianapolis, Indiana 46202
}

Received: March 5, 2003; In Final Form: June 2, 2003

\begin{abstract}
Monte Carlo simulations were used to investigate the adsorption of a polyelectrolyte chain on an oppositely charged micelle. The influence of the concentration of monovalent salt on the isolated polyelectrolyte chain was investigated by considering the chain expansion factor and persistence length. The polyelectrolytemicelle complex was characterized in terms of the amount of adsorbed polymer in trains, loops, and tails. The overcharging of the complex and the conformation of the adsorbed polyelectrolyte chain was also studied. The ionic strength adsorption-desorption limits were estimated at different micelle charge densities and compared to existing data for the experimental system of sulfonated poly(vinyl alcohol) and micelles of dimethyldodecylamineoxide of varying degrees of protonation. The effect of the relative micelle concentration on the adsorption-desorption limit of the system was also investigated.
\end{abstract}

\section{Introduction}

Complexation between polyelectrolyte chains, both synthetic and natural, and oppositely charged macroions such as organic and inorganic particles, proteins, micelles, and vesicles is of great interest in the fields of soft condensed matter, ${ }^{1,2}$ biology, ${ }^{3}$ environmental chemistry, ${ }_{4}$ and in the control of solution properties for industrial applications. ${ }^{5}$ Numerous applications are also expected to be found in the field of water treatment by flocculation. Nonetheless, the rational use of polyelectrolytes to control colloidal suspensions on the basis of the manipulation of long-range attractive and/or repulsive polyelectrolyte-colloid interactions via the chemical compositions, geometries, and relative concentrations of the different species is not well understood because the specific properties of polyelectrolyte and colloid solutions result in a multitude of complex structures and no clear picture covers the whole range of possible properties and structures, despite many theoretical and numerical studies. Using a combination of variational procedures and the ground-state dominance approximation as well as off-lattice Monte Carlo simulations, Von Goeler, Muthukumar, and Kong ${ }^{6,7}$ derived explicit equations for the dependence of the characteristics of the adsorption of polyelectrolytes onto curved surfaces (spheres and cylinders) on temperature, Debye screening length, polyelectrolyte charge density, molecular weight, and curvature. Adsorption was found to be promoted upon decreases in temperature, chain length, and salt concentration or upon increases in the radius and the surface charge density of the sphere. By considering the polyelectrolyte charge fraction and salt concentration, Haronska et al. ${ }^{8}$ proposed a theoretical model (based on a self-consistent approach) for the complex formalism of flexible polyelectrolytes and oppositely charged spheres. A particular emphasis on the influence of the finite size of both

\footnotetext{
† Part of the special issue "International Symposium on Polyelectrolytes".

* To whom correspondence should be sent. E-mail: serge.stoll@ cabe.unige.ch

$\doteqdot$ University of Geneva, http://www.unige.ch/cabe.

$\S$ Indiana-Purdue University.
}

polyelectrolytes and spheres was considered. The theoretical results were compared with the adsorption of PMA on a cationic micronetwork, and it was demonstrated that the criterion for critical adsorption shows different behavior for small and large curvatures of the sphere.

The recent scaling theory of Schiesse ${ }^{9}$ yields an approximate treatment of the chain-sphere complex over the whole range of parameters and especially fills the gaps that were left open by the existing theories and simulations. It permits the identification of a few independent scaling parameters in this system and leads to the construction of 2D phase diagrams (one for short and one for long screening lengths) that cover the whole range of all the other parameters.

Computer simulations allow approximate treatments of polyelectrolyte/sphere complexes over a large range of parameters and in some situations fill the gap between existing theories and experiments. Chain flexibility, linear charge density, and micelle radius were considered by Wallin and Linse ${ }^{10-12}$ to investigate the behavior of a polyelectrolyte-micelle complex. The complexation between a linear flexible polyelectrolyte and several oppositely charged macroions was recently examined by Jonsson and Linse ${ }^{13,14}$ by focusing on the effect of linear charge density, chain length, macroion charge, and chain flexibility. Chodanowski, Laguecir, and Stoll ${ }^{15-18}$ investigated the adsorption of flexible and semiflexible chains on spheres by considering curvature effects and the overcharging issue and by calculating adsorption limits. Recent studies by Anachina and Linse ${ }^{19}$ focused on the complexes between several chains and spheres. ${ }^{20}$ Nonetheless, very few studies have been undertaken to model a specific system and compare numerical modeling with experimental measurements.

In this work, we use Monte Carlo simulations to model the adsorption of a polyanion chain constituted of partially sulfonated poly(vinyl alcohol) (PVAS) on an oppositely charged dimethyldodecylamine oxide (DMDAO) micelle whose charge density can be controlled by $\mathrm{pH}$ via protonation of the surfactant headgroup. Thermodynamic as well as kinetic factors controlling 
the stability of these mixtures have been addressed experimentally by Dubin and co-workers. ${ }^{20}$ Critical conditions for adsorption versus $\mathrm{pH}$ and ionic concentration were reported, showing that the required critical surface charge density of the micelles necessary for adsorption was proportional to the inverse Debye screening length $\kappa$.

A coarse-grained model with a uniformly charged hard sphere to mimic a micelle particle and a pearl necklace chain consisting of point charges connected to each other are used. The model is parametrized to represent the main and pertinent physicochemical system characteristics. A Debye-Hückel approach, which represents an established level of description, is used (i.e., ions and counterions are implicitly considered). The adsorption/ desorption limit that is an important measurable parameter is investigated and compared to the experimental data. The polyelectrolyte conformations are analyzed prior to and after adsorption by considering the polymer structure at the interface (number of monomers in trains, loops and tails). Equilibrated conformations are also provided to achieve qualitative pictures of the polymer/micelle complexes. The adsorption of a polyelectrolyte on a colloid micelle is presented here with special attention focused on the effects of the surface charge density of the micelle and salt concentration on the adsorption/desorption limit. Polyelectrolyte-micelle complex conformation and chargeinversion phenomena as well as the effect of the chain persistence length and the effect of the relative micelle concentration on the adsorption-desorption limit of the system are also studied.

\section{Experimental System}

The modeled experimental system involves the binding of nonionic/cationic DMDAO micelles to oppositely charged polyanions of variable charge density, namely, poly(vinyl alcohol) of varying degrees of sulfation. Turbidimetry and dynamic light scattering show complexation occurring at a welldefined critical $\mathrm{pH}_{\mathrm{c}}$ corresponding to a critical micelle surface charge density that, for any degree of sulfation, depends uniquely on ionic strength $I$, and $\sigma$ was so investigated. Depending on $\mathrm{pH}$ and $I$, DMDAO micelles may have either spherical or cylindrical geometry. Here, only the spherical micelle regime is considered. In addition to having simple geometry and readily controllable charge densities, DMDAO micelles have clearly defined micelle surfaces because of the small size of the headgroup. The titratable headgroup also allows the direct measurement of the micelle surface potential without supplemental probe molecules. Consequently, the micelle may be considered to be a uniformly charged hard sphere with different charge density, and the polyelectrolyte chain is modeled as a pearl necklace chain consisting of interconnected point charges.

The DMDAO concentration is $1 \times 10^{-2} \mathrm{M}$; because the aggregation number of DMDAO under these conditions is close to $100,{ }^{21}$ this corresponds to a micelle concentration of $1 \times$ $10^{-4} \mathrm{M}$. The experimental PVAS chain concentration is $2.5 \times$ $10^{-5} \mathrm{M}$, so the experimental stoichiometric ratio between micelles and PVAS chains is 4.

Simulations are carried out on a system composed of one micelle and one polyelectrolyte chain in a box. As the box volume is taken into account, we can calculate the micelle and the chain concentrations that are respectively the same: $1.28 \times$ $10^{-6}$ moles of micelles per liter or $1.28 \times 10^{-6}$ moles of chains per liter. The concentration of this diluted system is more or less equivalent to that in the experiments. To study the effect of the relative micelle concentration on the adsorption- desorption limit, we also performed simulations under the experimental stoichiometric conditions.

\section{Computational Section}

Model and Method. Polyelectrolytes are represented here so as to model a class of material by a succession of $N$ freely jointed hard spheres or physical monomers of radius $\sigma_{\mathrm{m}}$. Hence, a coarse-grained approach is used. The fraction of ionized monomers $f$ is adjustable. The bond length and the intercharge distance are constant. (See the Model Parametrization section.)

The micelle is represented as an impenetrable, uniformly charged sphere with a constant radius of $\sigma_{\mathrm{p}}$. The micelle surface charge is assumed to be concentrated in a charged point located at its center and adjusted so as to kept a constant surface charge density $\sigma$ that is representative of the surface charge density of the micelles.

The solvent is treated as a dielectric medium with a relative dielectric permittivity constant $\epsilon_{\mathrm{r}}$ taken as that of water at 298 $\mathrm{K}$ (i.e., 78.5). The total energy $E_{\text {tot }}$ (in $k_{\mathrm{B}} T$ units) for a given conformation is the sum of repulsive electrostatic interactions between monomers, attractive electrostatic interactions between chains, and the particle $E_{\text {el. }}$ Hard-core interactions $E_{\text {ev }}$ are also considered to include both excluded volumes. All pairs of charged monomers within the polyelectrolyte interact with each other via a screened Debye-Hückel long-range potential,

$$
u_{\mathrm{el}}\left(r_{i j}\right)=\frac{z_{i} z_{j} e^{2}}{4 \pi \epsilon_{\mathrm{r}} \epsilon_{0}\left(r_{i j}+\zeta\right)} \exp \left(-\kappa\left(r_{i j}+\zeta\right)\right)
$$

where $z_{i}$ represents the amount of charge on unit $i$, and $r_{i j}$ represents the distance between two monomers $i$ and $j$. $\zeta$ represents an additional term for the distance between monomers to take into account the delocalization of the charges from the chain backbone (Figure 1).

Monomers interact with the particle according to a VerweyOverbeek potential,

$$
u_{\mathrm{el}}^{\prime}\left(r_{i j}\right)=\frac{z_{i} z_{\mathrm{j}} e^{2}}{4 \pi \epsilon_{\mathrm{r}} \epsilon_{0} r_{i j}} \cdot \frac{\exp \left[-\kappa\left(r_{i j}-\sigma_{\mathrm{p}}\right)\right]}{1+\kappa \sigma_{\mathrm{p}}}
$$

Free ions are not included explicitly in the simulations, but their overall effects on monomer-monomer and monomer-particle interactions are described via the dependence of the inverse Debye screening length $\kappa$ on the electrolyte concentration.

Monte Carlo (MC) simulations are performed according to the Metropolis algorithm in the canonical ensemble. In this method, successive "trial" chain configurations are generated to obtain a reasonable sampling of low-energy conformations. After applying elementary movements that are randomly selected, the Metropolis selection criterion is employed either to select or reject the move. The perturbation process is continued a specified number of times (a typical run requires several millions of perturbations) until the conformation is energy-minimized and equilibrated. To generate new conformations, the monomer positions are randomly modified by specific movements. These movements include three "internal" or elementary movements (end-bond, kink-jump, and crankshaft), the pivot, and the reptation. ${ }^{22}$

Model Parametrization. The PVAS chain with $M_{\mathrm{w}(\mathrm{PVAS})}=$ $20000 \mathrm{~g} / \mathrm{mol}$ corresponds to an average degree of polymerization $N=454$. We have carried out molecular dynamics calculations using Cerius2 (UFF Universal Force Field 1.02). ${ }^{23}$ The geometry of a small polymer containing 20 monomers has been optimized, and the distributions of carbon-carbon 


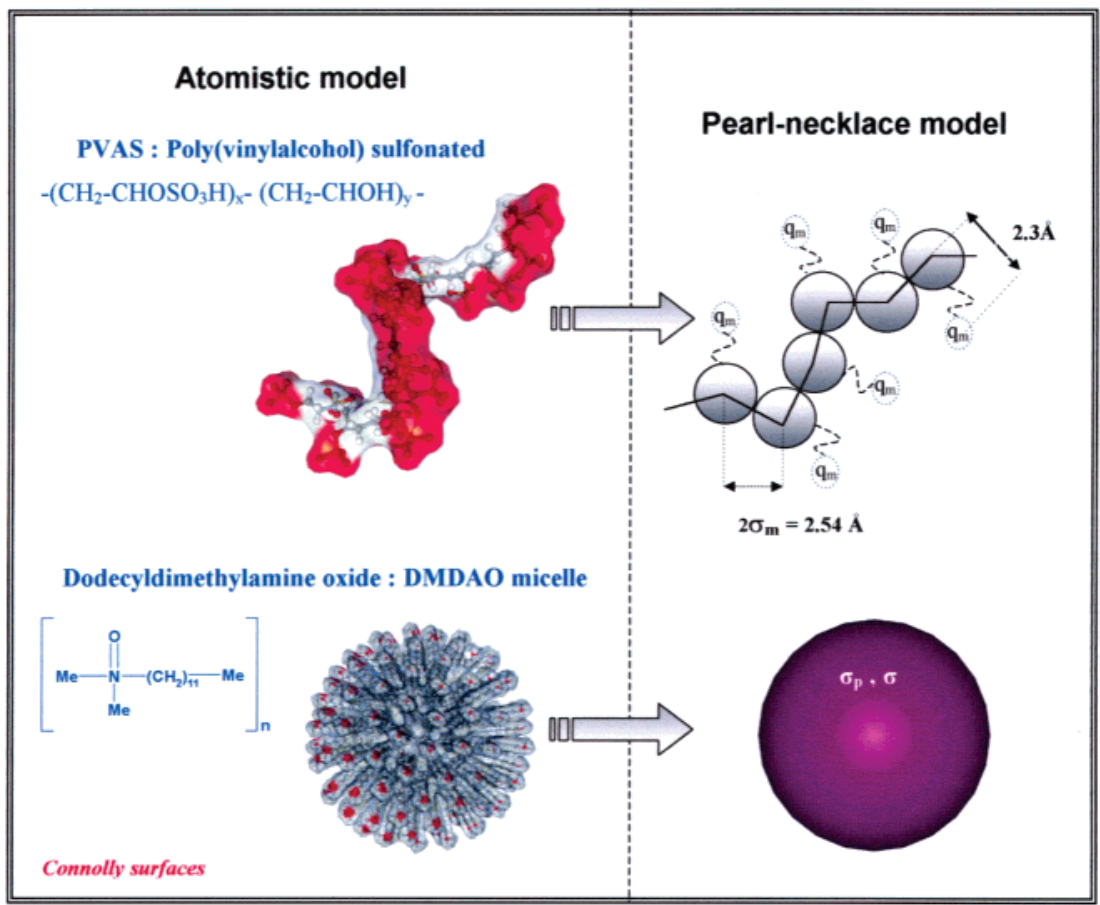

Figure 1. Coarse-grain model and equivalent atomistic description of the DMDAO-PVAS system. $\left(q_{\mathrm{m}}\right.$ is the monomer charge equal to 0 or $-1 e$ according to the linear charge density of the chain $f$, which is equal to 0.8 ; charge delocalization was evaluated as $2.3 \AA$ from the monomer center; $\sigma_{\mathrm{m}}$ is the monomer radius equal to $1.27 \AA$; the number of monomer is $N=454$.) ( $\sigma_{\mathrm{p}}$ is the micelle radius equal to $25 \AA$, and $\sigma$ is the micelle surface charge density varying from 4.5 to $200.4 \mathrm{mC} \cdot \mathrm{m}^{-2}$ ).

$(\mathrm{C}-\mathrm{C})$ and sulfur-sulfur $(\mathrm{S}-\mathrm{S})$ distances have been calculated. The mean monomer-monomer distance based on $\mathrm{C}-\mathrm{C}$ distances is relatively rigid and is equal to $2.54 \AA$. The corresponding chain contour length with $N=454$ is thus equal to $L_{\mathrm{c}}=1153 \AA$. The charge $q_{\mathrm{m}}$ of the charged monomers is set equal to $-1 e$, and the distance between the charges is calculated by considering the distance between monomers and the charge delocalization. With $80 \%$ sulfation of PVA polymer in the experiment, $f$ is set equal to 0.8 . The delocalization of the charge on the sulfonated groups from the chain backbone was evaluated as $2.3 \AA$. Hence $\zeta$, the delocalization parameter, was set equal to $4.60 \AA$ (i.e., $2 \times 2.3 \AA$ ). During the molecular dynamic simulations, the mean distance between successive monomer charges was found to be roughly the Bjerrum length, $l_{\mathrm{B}}=7.14$ $\AA$.

The DMDAO micelle in the spherical regime is represented as a particle with radius $\sigma_{\mathrm{p}}=25 \AA$. The central point charge $Q$ is adjusted from 2.2 to $100 e$ to set the micelle charge density $\sigma$ in the range of 4.5 to $204 \mathrm{mC} \cdot \mathrm{m}^{-2}$.

\section{Results and Discussion}

Isolated Flexible Chain. We first present conformations of isolated polyelectrolyte chains. These equilibrated conformations are used as initial conformations in the micelle-polyelectrolyte complex calculations. Ionic strength from the electrolyte is adjusted from $I=0 \mathrm{M}\left(\kappa^{-1}=\infty\right)$ to $I=2 \mathrm{M}\left(\kappa^{-1}=2.14 \AA\right)$, covering the range from the salt-free limit to the highly screened limit. Equilibrated structures are extracted from MC calculations and presented in Figure 2 versus $I$. As shown in Figure 2, polyelectrolyte conformations are linked to the intensity of the electrostatic repulsions between the monomers. Strong electrostatic interactions promote the formation of extended chains having significant persistence lengths.

The mean-square radius of gyration $\left\langle R_{\mathrm{g}}{ }^{2}\right\rangle$ and the mean-square end-to-end distance $\left\langle R_{\mathrm{ee}}{ }^{2}\right\rangle$ were calculated to derive the expan-

\begin{tabular}{|c|c|c|c|}
\hline$\kappa^{-1}[\AA]$ & $\boldsymbol{I}[\mathrm{M}]$ & $\begin{array}{l}\text { Snapshots of } \\
\text { equilibrated } \\
\text { conformations }\end{array}$ & $\begin{array}{c}\text { Total Persistence } \\
\text { length } \\
\mathbf{L}_{\text {p,cal }}[\AA]\end{array}$ \\
\hline$\infty$ & 0 & & 7812.5 \\
\hline 30.30 & 0.01 & & 432.9 \\
\hline 15.15 & 0.04 & & 177.3 \\
\hline 9.58 & 0.1 & & 129.5 \\
\hline 4.28 & 0.5 & & 73.2 \\
\hline 2.14 & 2 & & 53.6 \\
\hline
\end{tabular}

Figure 2. Snapshots of equilibrated conformations and the corresponding total persistence length $L_{\mathrm{p}, \text { cal }}$ (orientational correlation functions) at different Debye lengths.

sion factor

$$
r=\frac{\left\langle R_{\mathrm{ee}}{ }^{2}\right\rangle}{\left\langle R_{\mathrm{g}}{ }^{2}\right\rangle}
$$

which quantifies the conformational changes of the polyelec- 

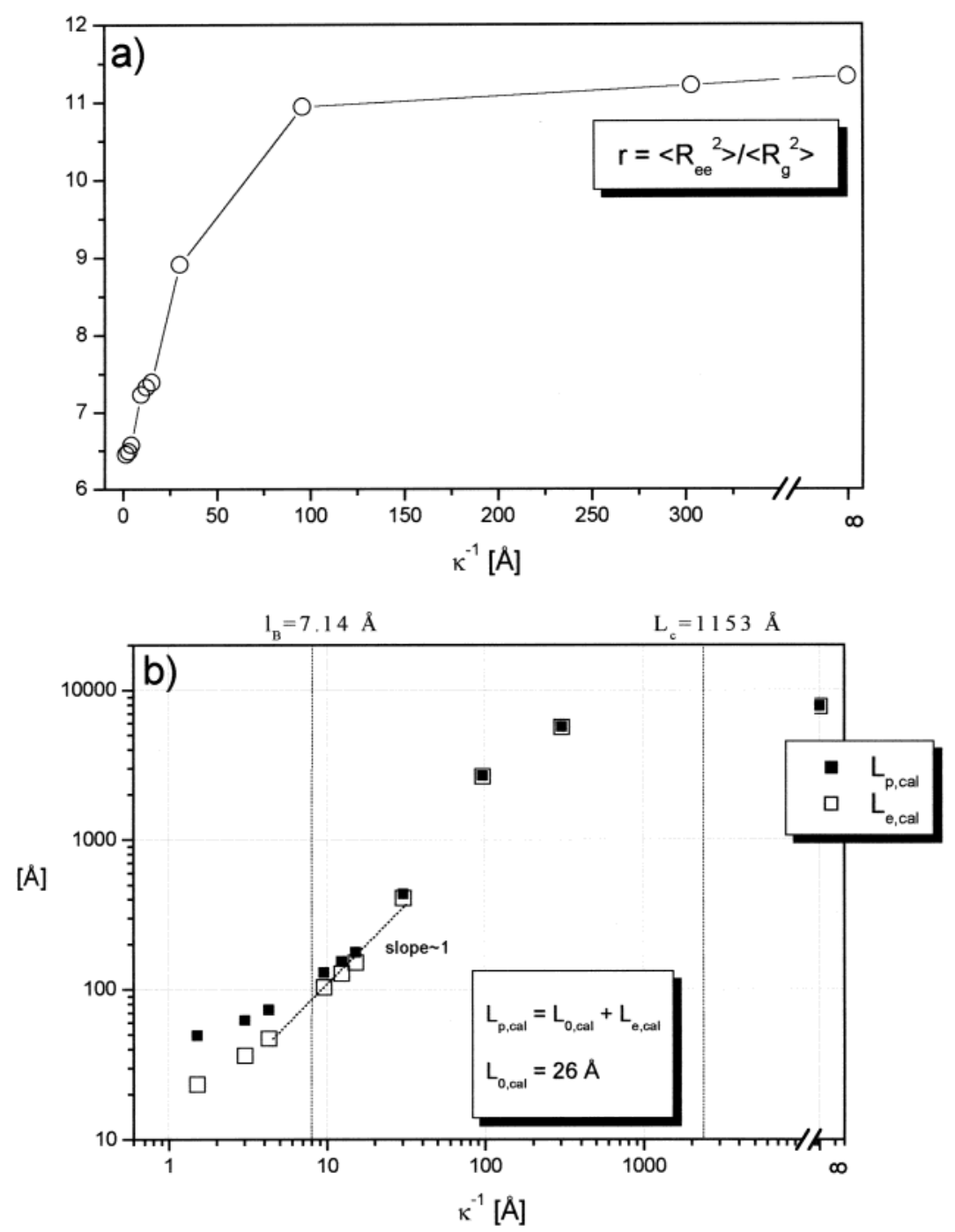

Figure 3. (a) Expansion factor $r=\left\langle R_{\mathrm{ee}}{ }^{2}\right\rangle\left\langle\left\langle R_{\mathrm{g}}{ }^{2}\right\rangle\right.$ of the isolated flexible chain versus the Debye length. $r=12$ corresponds to a rigid rod, and $r=$ 6 corresponds to a random walk. The expansion factor $r$ evolved from 6.5 at low Debye length to 11.3 at high Debye length, corresponding to a stretching of the chain. (b) Representation of the persistence length of the isolated flexible chain versus the Debye length. (The contour length of the chain is $1153 \AA$.) The total persistence length of the chain $L_{\mathrm{p}, \text { cal }}$ is the sum of an intrinsic contribution $\left(L_{0, \text { cal }}\right)$ and an electrostatic contribution $\left(L_{\mathrm{e}, \text { cal }}\right)$.

trolyte (Figure 3a). When the Debye screening length $\kappa^{-1}$ is less than the distance between two charges, the polymer assumes self-avoiding walk dimensions, and the expansion factor $r$ is close to 6.5. At high $\kappa^{-1}$, repulsions among the monomers have increased, resulting in extended structures with an expansion factor $r$ close to 11.3. This value is a little smaller than the predicted $r=12$ value for the rodlike conformations ${ }^{24}$ because of the thermal fluctuations during the Monte Carlo procedure that locally affect the monomer distribution.

The persistence length $L_{\mathrm{p}}$ is frequently used for experimental characterization of polymer chain comformation. The total persistence length of a polyelectrolyte $L_{\mathrm{p}}$ is the sum of an intrinsic contribution $\left(L_{0}\right)$ and an electrostatic contribution $\left(L_{\mathrm{e}}\right)$ :

$$
L_{\mathrm{p}}=L_{0}+L_{\mathrm{e}}
$$

The simplest and most natural definition for the persistence length is purely geometrical and is given by the expression for $L_{\mathrm{p}, \mathrm{cal}}$, which is calculated from the function of the correlation of the angles of connection (CAL). That function is defined as the scalar product of two normalized vectors (monomer to adjacent monomer) of the chain,

$$
\operatorname{CAL}(k)=\left\langle\frac{1_{i}}{l} \cdot \frac{1_{i+k}}{l}\right\rangle=\left\langle\cos \theta_{i, i+k}\right\rangle
$$

where $i$ and $k$ are vectors and $\theta_{i, i+k}$ is the angle between the two vectors that are considered. Because the persistence length is defined as the length along the chain after which any angular correlation disappears, the CAL function must present an exponential decay that is similar to that of the vermicular chains to which it applies:

$$
\operatorname{CAL}(k) \approx \exp \left(-\frac{k}{L_{\mathrm{p}, \mathrm{cal}}}\right)
$$

This can be expressed in logarithmic form as

$$
C(k)=\ln \left\langle\cos \theta_{i, i+k}\right\rangle \approx \ln \left\{\exp \left(-\frac{k}{L_{\mathrm{p}, \mathrm{cal}}}\right)\right\}=-\frac{k}{L_{\mathrm{p}, \mathrm{cal}}}
$$

allowing the calculation of $L_{\mathrm{p}, \mathrm{cal}}$ directly from the slope of $\mathrm{C}(k)$ versus $k$. Figure $3 \mathrm{~b}$ presents the $L_{\mathrm{p}, \mathrm{cal}}$, intrinsic $L_{0, \text { cal }}$, and 
TABLE 1: MC Equilibrated Conformations of the Polyelectrolyte/Micelle Complex versus the Ionic Concentration $I$ [M] and for Various Micelle Charge Densities $\sigma\left[\mathrm{mC} \cdot \mathrm{m}^{-2}\right]^{a}$

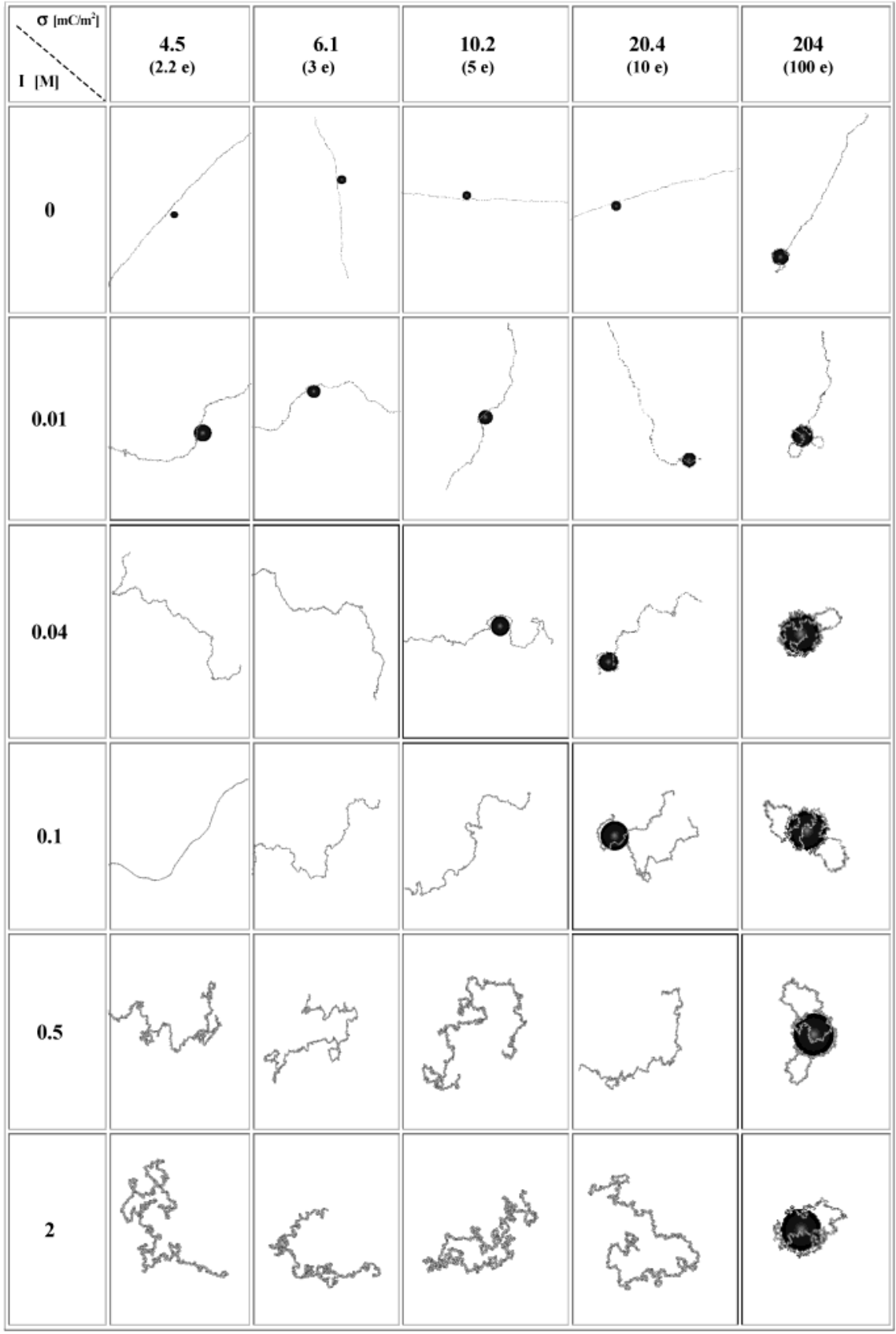

${ }^{a}$ The adsorption-desorption limit of the polyelectrolyte/micelle system is clearly observable and connected to the variation in ionic concentration and micelle charge density.

electrostatic $L_{\mathrm{e}, \mathrm{cal}}$ persistence lengths obtained from eq 6. $L_{0, \text { cal }}$ is obtained by considering a neutral chain.

The electrostatic persistence length exhibits four distinct regimes as a function of Debye length. At small salt concentrations, when $\kappa^{-1}>L_{\mathrm{c}}$ (the contour length), $L_{\mathrm{e}, \mathrm{cal}}$ is nearly constant. When the screening length is about the contour length, $\kappa^{-1} \approx L_{\mathrm{c}}, L_{\mathrm{e}, \text { cal }}$ is in a transition regime. After this transition, $L_{\mathrm{e}, \text { cal }}$ has a linear dependence on $\kappa^{-1}$ in the range from $\kappa^{-1} \approx$ $L_{\mathrm{C}} / 10$ to $\kappa^{-1}=l_{\mathrm{B}}$, and the slope is equal to 1 . A fourth regime exists when $\kappa^{-1}<l_{\mathrm{B}}$, where the chain is flexible, although this flexibility is limited by the segment length.

Micelle-Polyelectrolyte Interaction. An equilibrated polyelectrolyte chain and a spherical charged micelle are confined in a spherical box without contact with each other. Then the simulated system is allowed to relax for equilibration. Snapshots of equilibrated structures of polyelectrolyte/micelle complexes are represented as a function of micelle surface charge density $\sigma$ and ionic strength $I$ (Table 1). Because of the lack of available space, different scales have been used to represent the complexes. (The micelle diameter is constant.)

Adsorption is always observed in the low-salt regime $(I=0$ and $0.01 \mathrm{M}$ ), but when the ionic strength is further increased, chain desorption is achieved at low micelle charge surface density. The adsorption/desorption limit is hence sensitive both to the surface charge density $\sigma$ and ionic strength $I$. Low $\sigma$ and high $I$ values promote chain desorption.

A variety of adsorbed conformations can be observed. In the salt-free regime, two conformations are observed on the basis of the position of micelle-chain contact. Chains are mainly extended because of the unscreened monomer electrostatic 


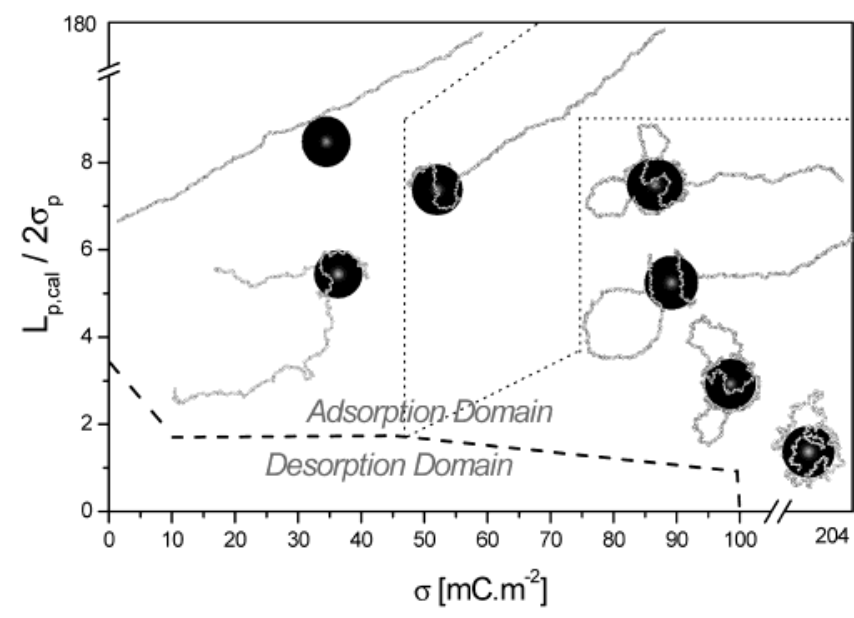

Figure 4. Diagram of conformational states for the micelle-chain complex as a function of the total persistence length of the isolated chain divided by the micelle diameter $L_{\mathrm{p}, \mathrm{cal}} / 2 \sigma_{\mathrm{p}}$ and the surface charge density of the micelle $\sigma$.

repulsions. At low $\sigma$ values, the micelle is tangential to the chain and is situated in its middle, whereas for the highest $\sigma$ the chain is wrapped around the micelle and a long tail is formed in solution (tadpole). At intermediate salt concentrations (from $I=0.01$ to $0.5 \mathrm{M}$ ), increasing $\sigma$ first promotes the adsorption of the chain on the micelle, followed by polyelectrolyte collapse onto the micelle surface. In addition to the conformations described above, additional structures are obtained at high micelle charge density $\sigma\left(204 \mathrm{mC} \cdot \mathrm{m}^{-2}\right)$ : first, when $I=0.01$ $\mathrm{M}$, loops appear in addition to one tail, and when $I=0.04 \mathrm{M}$, the tail disappears and loops decrease to form a tight complex where the chain is wrapped around the micelle. By further increasing the ionic concentration, conformations composed of large loops (without tails) called "multileafed rosettelike complexes" according to Schiessel et al. ${ }^{9}$ are formed, The formation of such complexes is due to steric hindrance (excluded volumes of monomers) at the surface of the micelle and the relatively small surface available for chain adsorption. We observed no loops when using smaller chains
In Figure 4 , the reduced persistence length $L_{\mathrm{p}, \mathrm{cal}} / 2 \sigma_{\mathrm{p}}$ and micelle charge density are used to indicate a micelle-polyelectrolyte complex phase diagram. The thick dotted line denotes the adsorption/desorption limi,t and the thin dotted lines denote the passage with increasing $\sigma$ from tangent conformations, tadpoles, and rosette structure as described by Schiessel et al. ${ }^{9}$

Adsorption-Desorption Limit. At a given simulation time, a polyelectrolyte conformation is defined as "adsorbed" when at least one monomer is positioned within one segment length (2.54 $\AA$ ) from the micelle surface for a given simulation run, and the polyelectrolyte is arbitrarily considered to be adsorbed when the number of adsorbed conformations is $>50 \%$. For a chosen $\sigma$ value, structures were obtained at various $\kappa$ 's to determine the critical Debye length (equivalently, a critical ionic strength). In Figure 5, we have compared the experimental and calculated critical Debye length and critical ionic strengths at different micelle surface charge densities $\sigma$. The comparison is limited to the experimental domain of spherical DMDAO micelles. It can be clearly seen that the adsorption/desorption limit is dependent both on micelle charge density and ionic strength, the limiting Debye length decreasing monotonically with increasing micelle charge density. Adsorption is thus promoted by increasing micelle surface charge density and decreasing ionic strength. The adsorption-desorption limits obtained with MC simulations are in reasonable agreement with the experimental data. Nevertheless, our model underestimates the strength of absorption observed in the experiment. At low micelle charge density, the simulated data are close to the experiment, but the gap between simulations and experiments increases with $\sigma$.

Differences in the adsorption/desorption limits between the simulations and experiment may reflect the difficulty in assigning an adsorption criterion that matches the critical adsorption in experiments. Part of the problem may stem from the critical absorption not being a sharp transition, as indicated by ultracentrifuge filtration studies of Feng. ${ }^{25}$ Refinements to the model may also be needed. In contrast to the single-molecule simulations, the experimental detection of complexation requires an excess of micelles per polymer chain. Accordingly, simulations were performed to gain insight into the effect of the micelle/ chain relative concentration on the adsorption/desorption limit.

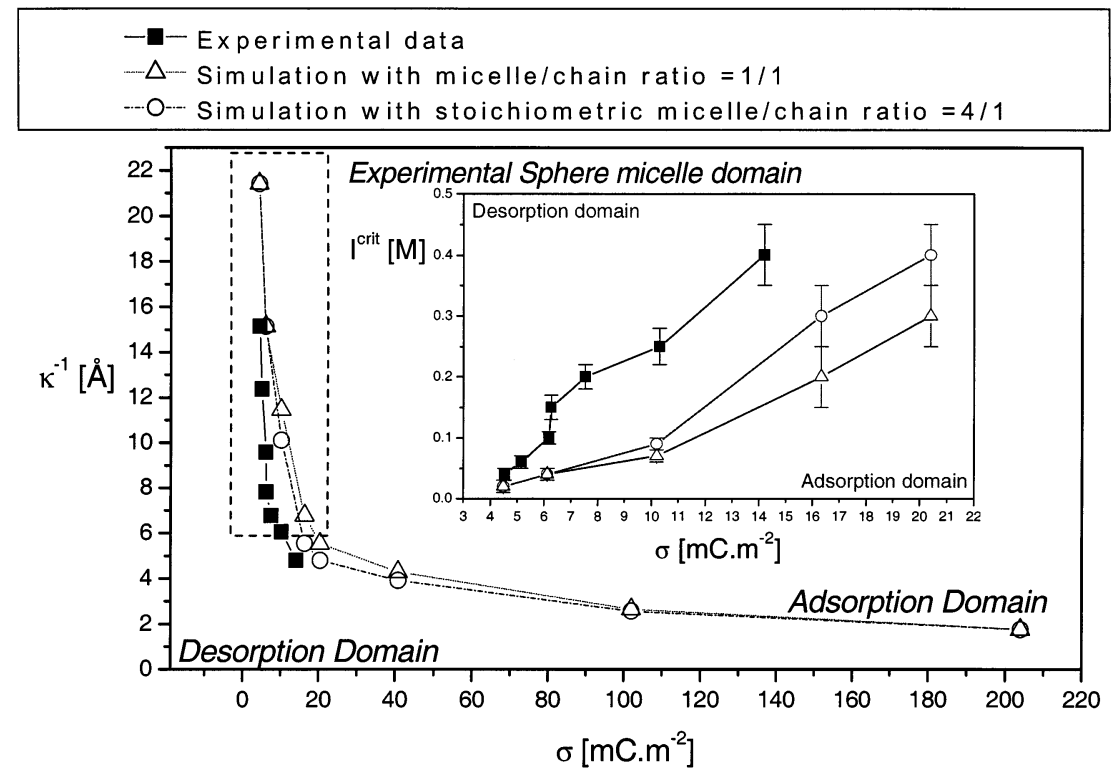

Figure 5. Comparison of the adsorption-desorption limit of the polyelectrolyte/micelle system computed by Monte Carlo simulations and corresponding experimental data (PVAS chain (poly(vinyl alcohol) partially sulfonated) on an oppositely charged DMDAO micelle (dimethyldodecylamine oxide) in the sphere regime. ${ }^{20}$ Monte Carlo simulations are obtained with two different micelle-to-chain ratios equal to 4 and 1. 
TABLE 2: Effect of the Micelle Concentration ${ }^{a}$

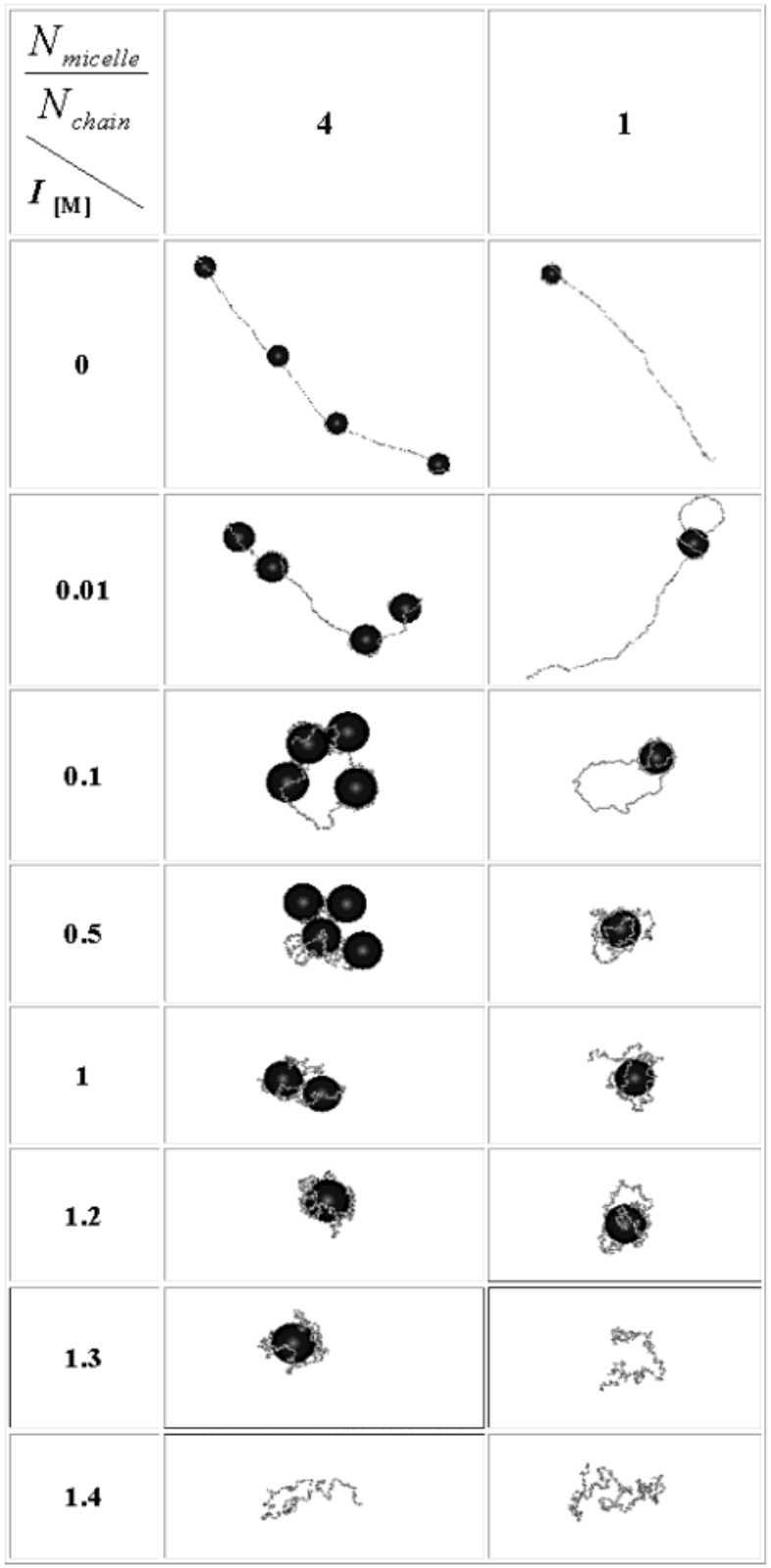

${ }^{a}$ Equilibrated structures of the polyelectrolyte/micelle complex represented as a function of ionic strength $I$ at a micelle surface charge density $\sigma$ equal to $102 \mathrm{mC} \cdot \mathrm{m}^{-2}$. Two relative micelle/chain concentration ratios are presented for comparison (a 1:1 ratio and a 4:1 ratio close to the stoechiometric ratio in the experiment).

Effect of the Relative Micelle Concentration. Simulations are now performed with a micelle/chain ratio of 4 , which is equal to the experimental stoichiometric ratio. In Table 2, snapshots of equilibrated structures of polyelectrolyte/micelle complexes at $4: 1$ and $1: 1$ stoichiometric ratios are represented as a function of the ionic strength for a micelle surface charge density $\sigma$ equal to $102 \mathrm{mC} \cdot \mathrm{m}^{-2}$.

At low salt concentration, the polyelectrolyte binds all four micelles by forming a linear complex in which micelles are separated by similar distances. With increasing ionic strength, the micelles are found at polyelectrolyte ends in dumbbell-like conformations, at which point micelles start to collapse to compact aggregates. Further increases in the salt concentration lead to progressive micelle desorption down to a 1:1 complex prior to full desorption at high ionic strengths. Noteworthy is the small but significant shift in the desorption limit from $I=$ $1.2 \mathrm{M}$ to $I=1.3 \mathrm{M}$ between the systems at the different ratios when $\sigma=102 \mathrm{mC} \cdot \mathrm{m}^{-2}$ because of the electrostatic repulsions between the micelles.

Hence, the adsorption/desorption limit is expected to be controlled by the relative micelle concentration. However, the effect of stoichiometry is shown in Figure 5 to be small, the shift between the simulation data appearing distinctly only at intermediate $\sigma$ densities. The adsorption-desorption limit is moved to higher ionic strengths for the more concentrated 4:1 system. At low $\sigma$, this shift is not present because the particles are weakly adsorbed and well separated and desorb simultaneously. At high $\sigma$, the strong attractive electrostatic interactions between the negatively charged polyelectrolyte and positively charged micelle encourage single-micelle adsorption at the same critical ionic strength. Then, because of the intermicellar repulsions, ionic strength has to be decreased to promote multiple micelle adsorption.

Conformation of the Adsorbed Polyelectrolytes. We investigated the conformational aspect of the adsorbed polyelectrolyte by calculating the number of trains, loops, and tails. In Figure $6 a-c$, the average number of monomers in trains, loops, and tails is presented as a function of the Debye length at different micelle charge densities $\sigma$.

Trains. The polyelectrolyte structure at the micelle surface can be derived by analyzing curves in Figure 6a. According to our definition of polymer adsorption, the absence of trains means that the polyelectrolyte is never adsorbed. In the other situations, below the critical value of $I$, a monotonic growth of monomers in trains is observed with increasing $\sigma$. At a given $\kappa^{-1}$ value, the number of monomers in trains increases with $\sigma$, corresponding to the flattening of the polyelectrolyte at the interface. It should be noted that the number of trains reaches a maximum at an intermediate ionic strength. This maximum value is achieved prior the formation of loops at the surface of the micelle.

Loops. According to Figure 6b, the global number of monomers in loops increases with $\sigma$. A maximum value is reached just prior to desorption. A smooth decrease with increasing $\kappa^{-1}$ is observed because monomers are transferred to trains or tails.

Tails. In Figure 6c, tail formation appears for all $\sigma$ when $\kappa^{-1}$ is high. The formation of tails is generally a sharp process, and most of the monomers are present as tails in the low-salt regime.

Chain Dimensions. When the polyelectrolyte is adsorbed on the micelle, its conformation is expected to be different from its conformation in solution without the influence of the micelle (isolated chain). Such a change can be described by the ratio $\left\langle R_{\mathrm{g}}{ }^{2}\right\rangle_{\text {ads }} /\left\langle R_{\mathrm{g}}{ }^{2}\right\rangle_{\text {free. }}$. This ratio is represented in Figure 7 versus the Debye screening length at various $\sigma$ values. Globally maximum deformation is achieved at intermediate salt concentration (i.e., when the chain adsorption is the most effective by considering the number of monomers in trains).

At small $\sigma$ and high Debye length $\kappa^{-1}$ values, the ratio $\left\langle R_{\mathrm{g}}{ }^{2}\right\rangle_{\text {ads }} /\left\langle R_{\mathrm{g}}{ }^{2}\right\rangle_{\text {free }}$ is close to 1 because the adsorption of the chain occurs tangentially, involving just a few monomers. When $\sigma$ is equal to $204 \mathrm{mC} \cdot \mathrm{m}^{-2}$ and when $\kappa^{-1}$ is large, the ratio $\left\langle R_{\mathrm{g}}{ }^{2}\right\rangle_{\text {ads }} l$ $\left\langle R_{\mathrm{g}}{ }^{2}\right\rangle_{\text {free }}$ is close to 0.4 because of the particular conformation adopted by the chain. (The micelle is adsorbed at the end of the chain, and the complex presents a long tail.) By decreasing $\kappa^{-1},\left\langle R_{\mathrm{g}}{ }^{2}\right\rangle_{\text {ads }} /\left\langle R_{\mathrm{g}}{ }^{2}\right\rangle_{\text {free }}$ decreases to reach a minimum at 0.05 and then increases again until it reaches 0.4. In that case, the $\left\langle R_{\mathrm{g}}{ }^{2}\right\rangle_{\text {ads }} l$ $\left\langle R_{\mathrm{g}}{ }^{2}\right\rangle_{\text {free }}$ value is directly connected to the emergence of loops 

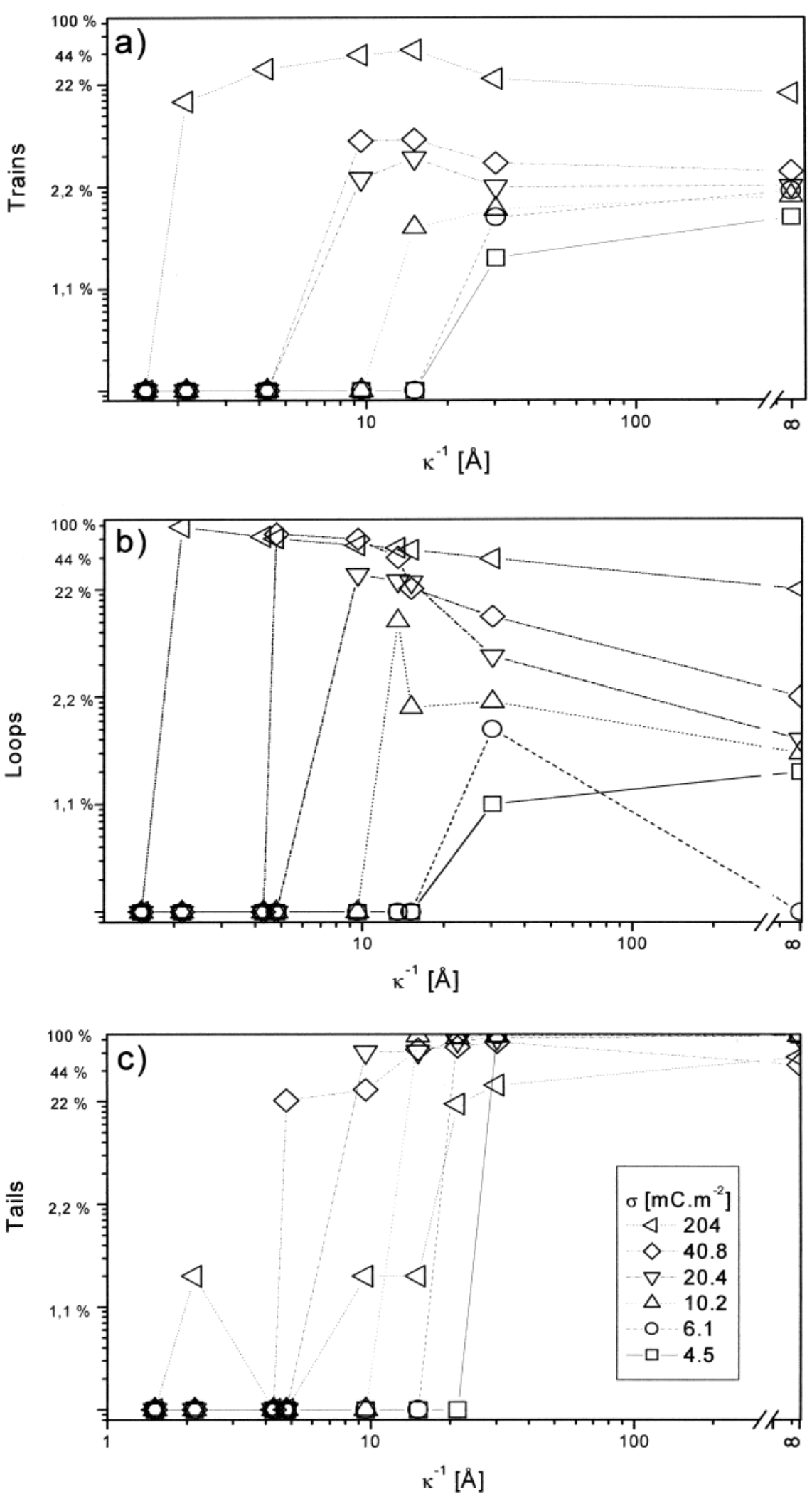

Figure 6. Number of monomers in trains (a), loops (b), and tails (c) as a function of the Debye length at different micelle charge densities $\sigma$ for the polyelectrolyte/micelle complex.

or the formation of multileafed rosettelike complexes from collapsed conformations.

Overcharging Phenomena. In this section, we study the total charge of the micelle-polyelectrolyte complex and therefore situations where charge inversion occurs (i.e., when the polyelectrolyte is sufficiently adsorbed with regard to the number of monomers in trains so that the net charge of the complex changes sign). In the system we investigated, the total number of charged monomers on the polyelectrolyte is equal to $360 e$, and the micelle charge $Q$ is adjusted from +2.2 to $+100 e$. Overcharging is calculated by considering the number of monomers in trains and the fraction of ionized monomers, which is defined by $Q^{*}=Q-\left(N_{\text {train }} * f \cdot e\right)$. Hence the ratio $\mathrm{Q} * / \mathrm{Q}$ represents the normalized charge of the complex. 


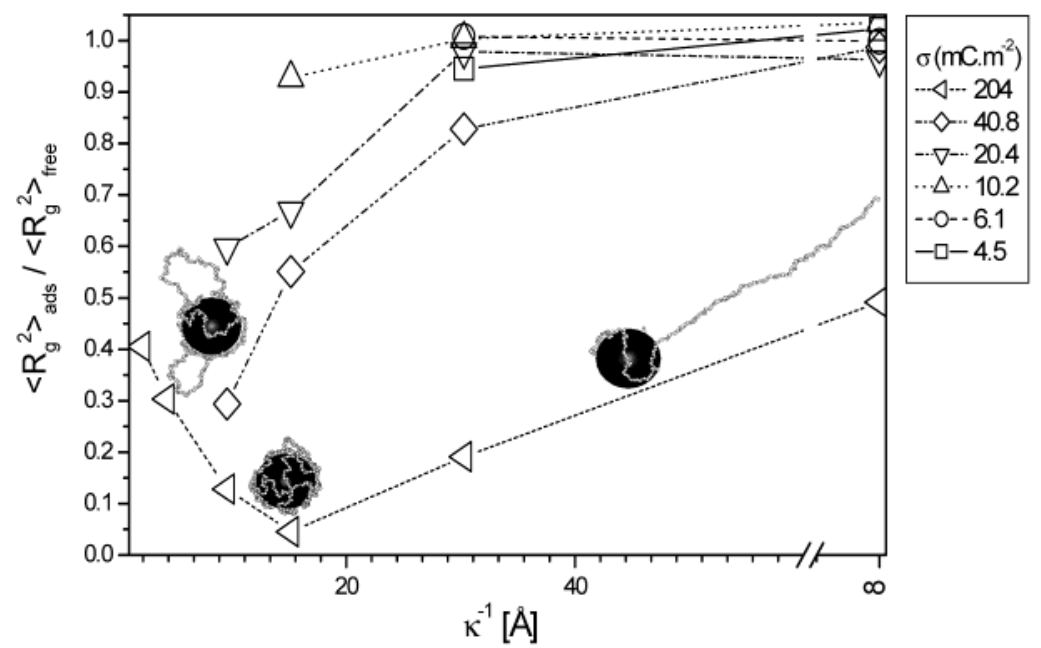

Figure 7. Ratio between the radius of gyration $R_{\mathrm{g}}$ ads of the adsorbed polyelectrolyte chain on the micelle and the radius of gyration $R_{\mathrm{g}}$ free of the corresponding free flexible polyelectrolyte chain versus the Debye length at different micelle charge densities $\sigma$.

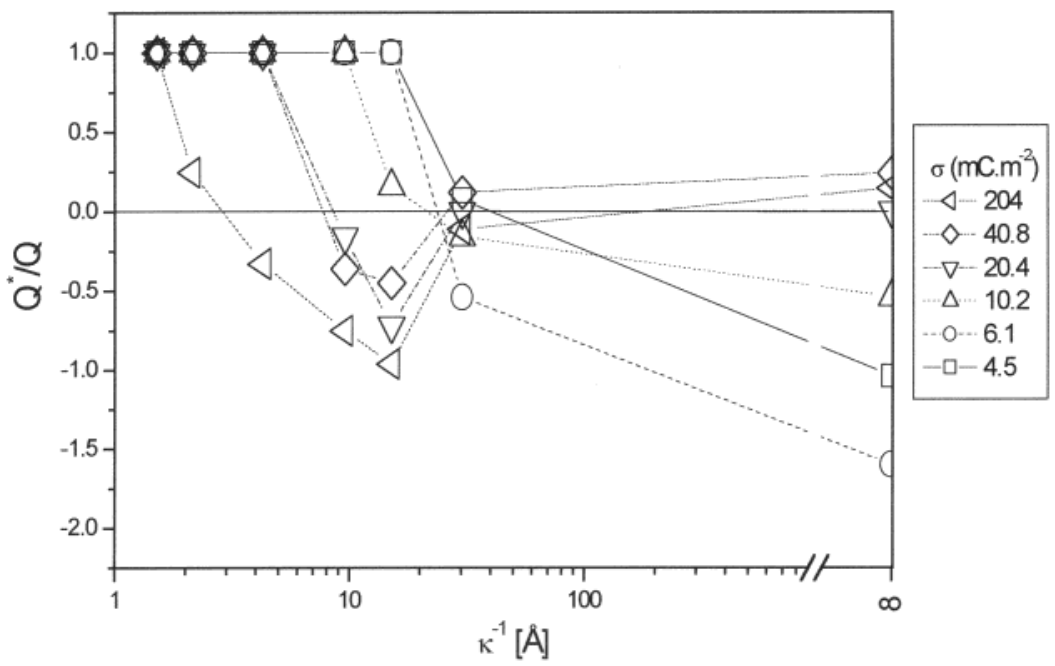

Figure 8. Normalized charge $Q^{*} / Q$ of the polyelectrolyte/micelle complex with respect to the Debye length at different micelle charge densities $\sigma$. The net charge of the polyelectrolyte/micelle complex $Q^{*}$ is defined by $Q^{*}=Q-\left(N_{\text {train }} * f \cdot e\right)$.

Figure 8 presents the normalized charge of the complex at different micelle charge densities varying from 4.5 to 204 $\mathrm{mC} \cdot \mathrm{m}^{-2}$ with respect to the Debye length $\kappa^{-1}$. We observe that the complex charge has nontrivial behavior because of the multitude of structures that are formed. For small $\sigma$ values, charge inversion occurs only for large $\kappa^{-1}$ values (twice the micelle charge at zero salt concentration). For the highest $\sigma$ values, charge inversion presents a maximum value at intermediate salt concentration and then decreases to reach charge neutralization. The presence of loops at high salt (i.e., rosette structures) concentration decreases the amount of adsorbed monomer in the train and hence reduces overcharging.

\section{Conclusions}

Monte Carlo simulations were used to study the interactions between a charged micelle and a flexible chain modeled according to a well-defined experimental system. We demonstrated that the complexation process leads to a multitude of structures ranging from collapsed complexes, tadpoles, and dumbbells to tangent conformations.

We found that the evolution of the adsorption-desorption limits found by MC simulations with respect to the charge density of the micelle and the salt concentration is in good agreement with the experimental data. Polyelectrolyte adsorption is promoted by increasing the attractive energy between the charged monomers and the oppositely charged micelle either by increasing the surface charge density of the micelle or decreasing the ionic concentration. The role of the relative micelle concentration was investigated, and it was shown that electrostatic repulsions between the micelles promote the formation of a 1:1 complex when the ionic strength is high. We also demonstrated that charge inversion has a maximum value at intermediate ionic strength according to the relative size used here between the micelle and polyelectrolyte persistence length.

At this point, our model seems to capture the physics of the interactions between linear polyelectrolytes and micelles with a regular surface charge distribution. Nevertheless, improving the description of the experimental system could be considered to make modeling more precise. Furthermore, refinements are presently under consideration to gain insight into the role of secondary effects by including explicit counterions and hydrophobic interactions, for example.

Acknowledgment. We express our thanks to Professor Jacques Buffle and Dr. Alain Porquet for stimulating discussions. We gratefully acknowledge the financial support received from the following sources: Swiss National Research Projects 2100-061750.00, Commission Suisse pour La Technologie et 
l'Innovation (CTI), Project Top Nano 215950.1 and 6056.2, and the BASF Corporation.

\section{Glossary}

$\begin{array}{ll}\left\langle R_{\mathrm{ee}}{ }^{2}\right\rangle & \begin{array}{l}\text { mean square end-to-end length } \\ \left\langle R_{\mathrm{g}}{ }^{2}\right\rangle\end{array} \\ L_{\mathrm{c}} & \begin{array}{l}\text { mean square radius of gyration } \\ \text { chain contour length } \\ L_{\mathrm{p}, \mathrm{cal}}\end{array} \\ \begin{array}{l}\text { total persistence length of the chain (orientational } \\ \text { correlation) }\end{array} \\ L_{\mathrm{e}, \mathrm{cal}} & \begin{array}{l}\text { electrostatic persistence length of the chain } \\ \mathrm{L}_{0, \mathrm{cal}}\end{array} \\ N & \text { intrinsic persistence length of the chain } \\ \kappa^{-1} & \text { number of monomers of the polyelectrolyte chain } \\ I & \text { Debye screening length } \\ r & \text { ionic concentration } \\ Q & \text { polyelectrolyte expansion factor } \\ Q^{*} & \text { charge of the micelle } \\ \sigma_{\mathrm{m}} & \text { net charge of the polyelectrolyte/micelle complex } \\ \sigma_{\mathrm{p}} & \text { monomer radius } \\ \sigma & \text { radius of the micelle particle } \\ f & \text { charge surface density of the micelle particle } \\ l_{\mathrm{B}} & \text { fraction of ionized monomers } \\ q_{\mathrm{m}} & \text { Bjerrum length } \\ \zeta & \text { charge of a monomer }\end{array}$

\section{References and Notes}

(1) Barrat, J. L.; Joanny, J. F. In Advances in Chemical Physics; Prigogine, I., Rice, S., A., Eds.; Theory of Polyelectrolyte Solutions; Wiley \& Sons: New York, 1996; Vol. 94.
(2) Flory P. J. Principles of Polymer Chemistry; Cornell University: Ithaca, New York, 1992.

(3) Xia, J.; Dubin, P. L. In Macromolecular Complexes in Chemistry and Biology; Dubin, P., Bock, D., Eds.; Protein-Polyelectrolyte Complexes; Springer-Verlag: Berlin, 1994; p 247.

(4) Buffle, J.; Wilkinson, K. J.; Stoll, S.; Filella, M.; Zhang, J. Environ. Sci. Technol. 1998, 32, 2887.

(5) Vermeer, A. W. P.; Leermakers, F.; Koopal, L. Langmuir 1997, $13,4413$.

(6) von Goeler, F.; Muthukumar, M. J. Chem. Phys. 1994, 100, 7796.

(7) Kong, C. Y.; Muthukumar, M. J. Chem. Phys. 1998, 109, 1522.

(8) Haronska, P.; Vilgis, T. A.; Grottenmuller, R.; Schmidt, M. Macromol. Theory Simul. 1998, 7, 241.

(9) Schiessel Helmut, arXiv: cond-mat/0208433, 2002; Vol. 1 p 22.

(10) Wallin, T.; Linse, P. Langmuir 1996, 12, 305.

(11) Wallin, T.; Linse, P. J. Phys. Chem. 1996, 100, 17873.

(12) Wallin, T.; Linse, P. J. Phys. Chem. B 1997, 101, 5506.

(13) Jonsson, M.; Linse, P. J. Chem. Phys. 2001, 115, 3406.

(14) Jonsson, M.; Linse, P. J. Chem. Phys. 2001, 115, 10975.

(15) Chodanowski, P.; Stoll, S. J. Chem. Phys. 2001, 115, 4951.

(16) Chodanowski, P.; Stoll, S. Macromolecules 2001, 34, 2320.

(17) Chodanowski, P.; Stoll, S. Macromolecules 2002, 35, 9556.

(18) Laguecir A.; Brynda M.; Stoll, S. Chimia 2002, 56, 702.

(19) Akinchina, A.; Linse, P. Macromolecules 2002, 35, 5183.

(20) Feng, X. H.; Dubin, P. L.; Zhang, H. W.; Kirton, G. F.; Bahadur, P.; Parotte, J. Macromolecules 2001, 34, 6373.

(21) Maeda, H. J. Phys. Chem. 1988, 92, 4490.

(22) Chodanowski, P.; Stoll, S. J. Chem. Phys. 1999, 111, 6069.

(23) Rappe, A. K.; Casewit, C. J.; Colwell, K. S.; Goddard W. A., III; Skiff, W. M. J. Am. Chem. Soc. 1992, 114, 10024 (Universal Force Field (UFF)).

(24) Stevens, M. J.; Kremer, K. J. Chem. Phys. 1995, 10, 1669.

(25) Feng, X.; Dubin, P. L. Langmuir 2002, 18, 2032. 